\title{
STRUCTURE OF A FLAME FRONT PROPAGATING AGAINST THE FLOW NEAR A COLD WALL
}

\author{
VADIM N. KURDYUMOV \\ E.T.S. de Ingeniería Industrial, \\ Universidad de Salamanca, Salamanca (Bejar), Spain \\ AMABLE LIÑÁN \\ E.T.S.I. Aeronáuticos, UPM, Madrid, Spain
}

\section{Introduction}

The effects of a cold wall in the propagation of flames in tubes has a long history, beginning with experiments carried out by Sir Humphrey Davy, early in the XIX century, at the Royal Institution; he showed that flame propagation was not possible for pipe diameters below a critical size. Flashback, or propagation of flame in tubes against the flow of the reactive mixture is more difficult, particularly if the mean velocity is larger than the velocity of propagation $S_{L}$ of a planar flame front.

A renewed interest in flashback has arisen recently, in connection with the development of new modes of combustion to reduce the levels of $N O_{x}$ emissions from gas turbine engines. One of the most promising schemes corresponds to the LP (Lean premixed) or LPP (Lean premixed pre-vaporized) combustors, where combustion takes place with lean premixed flames, in contrast with the traditional turbojet combustors based on diffusion controlled combustion; which leads to higher flame temperature and, thus, to higher $N O_{x}$ production. In LP or LPP burners, a lean mixture is generated, without combustion, in a premixing tube. The reaction should only take place, downstream, in the combustion chamber. These burners are prone to combustion instabilities in the combustion chamber, and also - due to the high temperature of reacting mixture associated with the high compression ratios to self-ignition in the premixing tube and also to flashback, or upstream propagation of the flame in this tube, which must be prevented from occurring.

The mean flow velocity in the premixing tube is large compared with $S_{L}$, so that flame flashback can only occur in two forms: first, when there is flow reversal in the tube, resulting from strong pressure oscillations in the combustion chamber due to combustion instabilities, see [Thibaut \& Candel, 1998]. Second, with flame propagating along the low velocity region, characterized by the velocity gradient $A$, of the boundary layer near the wall; there, the flame is strongly curved in the frame front region to become nearly parallel to the wall downstream. With an adiabatic wall the flame can reach the wall without quenching. However, in the more realistic 


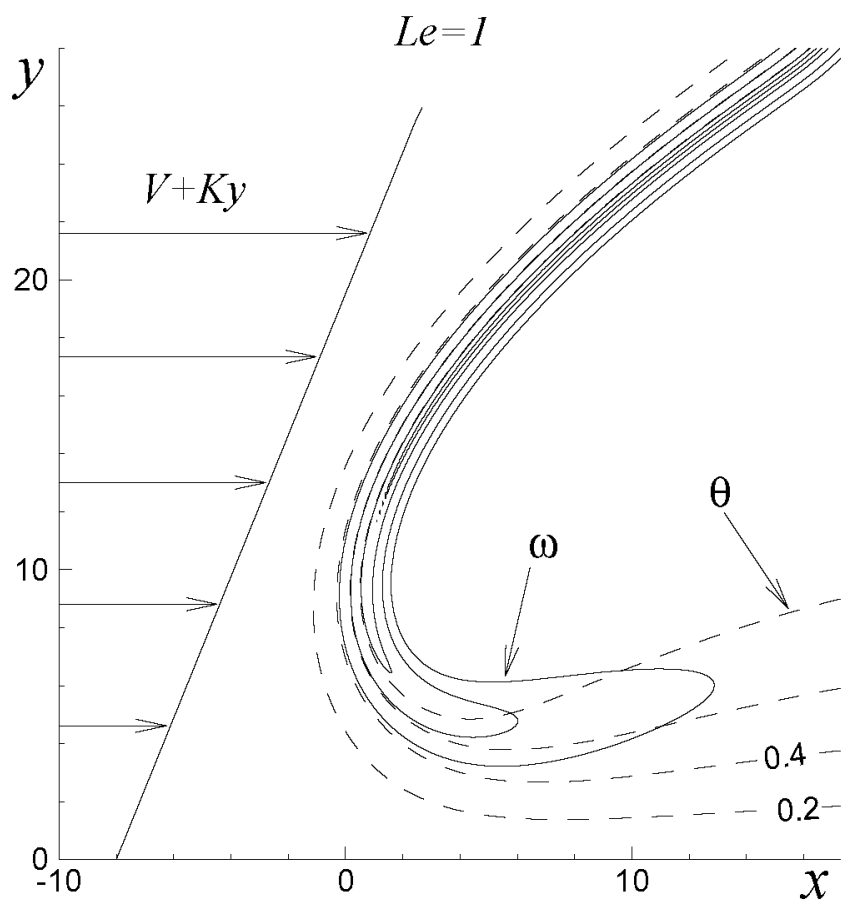

Fig. 1. Sketch of the velocity field, isotherms (dashed lines: $\theta$ at intervals 0.2 and $\theta_{\min }=0.2$ ) and reaction rate contours (solid lines: $\omega=0.01,0.1,1$ and 2 ) calculated at the onset of the flashback, $U / S_{L}=0.061, L e=1$ and $Z e=15$. The calculated value of the Karlovitz number is $K=0.061$.

case of an isothermal cold wall, with its temperature equal to the upstream flow temperature, the reaction is quenched near the wall, because of the low temperatures, and the flame is again bent downstream without reaching the wall. A typical flame front of this type is shown in Fig. 1.

A criterion of a critical value of $A_{c}$ of the velocity gradient below which flashback is possible, was put forward by Lewis and von Elbe [1943, 1951], based on the existence of a quenching layer, whose thickness depended on the reactive mixture. A pioneering study of the quench layer was carried out by Millán and von Karman [1953]. More detailed numerical studies were carried out much later beginning with the work of Lee and Tien [1982], who addressed the problem of flame propagation in tubes of small diameter, and the determination of the critical velocity gradient for flashback in tubes of large diameter. An experimental and numerical analysis of the onset flashback toward the slit and tubes feeding the reacting mixture to the combustion chamber of boilers for central heating is given by Mallens and Goey [1998].

More recently Kurdyumov et al. [2000] analyzed, and gave preliminary results for the prob- lem of flame flashback along the low velocity region of the boundary layer, to account for the effects of nonunity Lewis number of the limiting reactant on the flame front propagation velocity. The limiting reactant is the fuel in LPP burners, and the Lewis number $L e$ is the ratio of the thermal and fuel diffusivities. The effects of the Lewis number of the limiting reactant on the propagation of flames in tubes without flow of the reacting mixture have also been analyzed by Kurdyumov and Fernández [2002].

The present paper is devoted to the description of some of the drastic changes in flame front structure that occur, near a cold wall, due to the combined effects of the velocity gradient of the upstream flow and the difference from unity of the Lewis number.

It is well known that a planar flame front is unstable due to the density changes associated with the heat release (the Darrieus-Landau instability), and also due to nonequal diffusivities; see the reviews by Sivashinsky [1983] and Clavin [1985, 1994, 2000]. These instabilities, which lead to traveling waves and cellular structures, and eventually to chaotic flame behavior, are hindered by the flame front curvature, associated with the flame propagating in the shear flow near a wall. These stabilizing effects are particularly strong near the onset of flashback, when the front radius of the curvature, $l_{F}$, is of the order of the planar flame front thickness, $\delta_{L}$, as will be shown below.

Although the density changes due to the combustion heat release modify the flow field and, thereby, affect the flame structure, in this paper we shall neglect these changes; limiting our analysis to the effects of flame front curvature and nonunity Lewis number on the structure. Thus, we shall use the so-called thermal-diffusive model, for which the flow field is not effected by the heat release. In the flame front region, which determines the flame front propagation velocity relative to the wall, the velocity is then considered to be parallel to the wall with a constant velocity gradient $A$.

For simplicity in the presentation, the thermal diffusivity of the mixture, as well as the Lewis number, will be considered to be constant. The kinetics will be modeled as an overall Arrhenius reaction, with a rate characterized by the value of the planar flame velocity, $S_{L}$, and the Zeldovich number, $Z e$, or nondimensional activation energy to be defined below; which is typically large so that the reaction is frozen upstream from the flame front. 
Our aim is to determine, first, the conditions for a constant flame front propagation velocity $U$ relative to the wall, which when positive will indicate upstream propagation or flashback; when negative the front will be swept downstream by the flow. As we shall see below, steady flame front propagation will be encountered for fuels with realistic values of the Lewis number larger than unity; but not too large to encounter a pulsating mode of flame propagation. For lighter fuels, with a Lewis number close to unity or smaller than unity, we shall find multiplicity of steady solutions of the conservation equations for a range of values of the nondimensional velocity gradient, or Karlovitz number $K=A \alpha / S_{L}^{2}$.

In all cases the solution corresponding to flashback only exists for $K<K_{c}$. However, for $L e<$ 1 , we shall also find unsteady solutions, typically chaotic, of the conservation equations with a value $U$ of the front velocity variable with time. The resulting values of $U$ can be significantly large compared with $S_{L}$ if $K$, which is equal to the ratio $\delta_{L} / l_{F}$, is small compared with unity, when the effects of flame curvature are small.

It is a pleasure to contribute to this issue of Int. J. Bifurcation and Chaos, honoring Manuel G. Velarde, with an analysis of a problem of great technical relevance in which the key ingredients of nonlinearity play an important role; being responsible for the existence and possible multiplicity of traveling front solutions and their bifurcation to chaotic behavior. The senior author, A. Liñán, is indebted to Manuel for educating him very early in the importance and beauty of the subject.

\section{Mathematical Formulation}

As indicated before, the analysis will be based on the thermal-diffusive approximation, with the values of the density $\rho$, thermal diffusivity $\alpha$, constant pressure specific heat $c_{p}$ and diffusion coefficient $D$, assumed to be constant. The reaction is modeled by an irreversible overall reaction of the form

$$
F \rightarrow P+Q,
$$

where $F$ denotes the fuel, $P$ the products, and $Q$ the heat released per unit mass of fuel. The reaction rate $\Omega$, defined as the mass of fuel consumed per unit volume and unit time, is assumed to follow an Arrhenius law of the form:

$$
\Omega=\rho B Y \exp (-E / R T)
$$

where $B, Y, T$ and $E / R$ represent, respectively, the pre-exponential factor, mass fraction of the fuel, and the local and activation temperatures, respectively.

By neglecting the variations in density and viscosity of the gas, the velocity field is no longer affected by the temperature variation due to the release of heat. In the base of the laminar boundary layer, near the wall, the velocity of the fluid relative to the wall is given by $u=A y, v=0$; where $x$ and $y$ are distances along and normal to the wall, and $A$ is the constant velocity gradient at the wall.

It is convenient to formulate the problem in nondimensional form using scales derived from the well known structure of the steady planar laminar flame. See, for example [Williams, 1985]. In these flames $S_{L}$ is the flame velocity, given below, and $T_{e}=T_{0}+Q Y_{0} / c_{p}$, where $T_{0}$ and $Y_{0}$ are the initial temperature and concentration of fuel in the fresh mixture, is the so-called adiabatic flame temperature. The corresponding thickness $\delta_{L}$ of the preheated zone is of the order of $\delta_{L}=\alpha / S_{L}$.

To formulate the problem in a nondimensional form, we shall use $\delta_{L}$ and $\delta_{L} / S_{L}$ as scales for the length and time. Introducing the no-dimensional temperature, $\theta=\left(T-T_{0}\right) /\left(T_{e}-T_{0}\right)$, and using the initial value $Y_{0}$ for normalization of the fuel mass fraction, the governing equations, written for a reference frame moving upstream with a speed $U(t)=S_{L} V(t)$ relative to the wall, take the form

$$
\begin{gathered}
\frac{\partial \theta}{\partial t}+(V(t)+K y) \frac{\partial \theta}{\partial x}=\frac{\partial^{2} \theta}{\partial x^{2}}+\frac{\partial^{2} \theta}{\partial y^{2}}+\omega, \\
\frac{\partial Y}{\partial t}+(V(t)+K y) \frac{\partial Y}{\partial x}=\frac{1}{L e}\left(\frac{\partial^{2} Y}{\partial x^{2}}+\frac{\partial^{2} Y}{\partial y^{2}}\right)-\omega,
\end{gathered}
$$

where $\omega=\Omega \delta_{L} / S_{L} \rho Y_{0}$ is given by

$$
\omega=\frac{Z e^{2} Y}{2 L e u_{p}^{2}} \exp \left\{\frac{Z e(\theta-1)}{(1+\gamma(\theta-1))}\right\} .
$$

In this paper we consider the limiting case when the temperature of the wall is fixed equal to that of the unburned mixture, $T_{0}$. Thus, the wall boundary conditions are

$$
\theta=0, \quad \text { and } \quad \partial Y / \partial y=0 \text { at } y=0 .
$$

Far upstream and away from the wall

$$
\theta=0, \quad Y=1 \quad \text { at } \quad x \rightarrow-\infty \quad \text { or } \text { at } y \rightarrow \infty \text {. }
$$


Downstream of the flame front we require

$$
\partial \theta / \partial x \rightarrow 0, \quad \partial Y / \partial x \rightarrow 0 \quad \text { at } \quad x \rightarrow \infty
$$

which are satisfied by a downstream asymptotic structure, for $x \gg 1$, that can be described using the boundary layer approximation.

The following nondimensional parameters remain: the Lewis number $L e=\alpha / D$, the Zeldovich number $Z e=E\left(T_{e}-T_{0}\right) / R T_{e}^{2}$, assumed to be large compared with unity, the heat release parameter $\gamma=\left(T_{e}-T_{0}\right) / T_{e}$, and the Karlovitz number, defined as $K=\delta_{L} A / S_{L}$. The last one measures the stretching of the flame by the flow with the velocity gradient $A$. In what follows, anticipating that in the region where the chemical reaction term is important $Z e(\theta-1)$ is of order unity, the factor $\{1+\gamma(\theta-1)\}$ in (3) will be replaced by 1 , thus reducing the remaining nondimensional parameters to $K, L e$ and $Z e$.

Note that the factor $u_{p}=S_{L} / S_{a}$ arises in the nondimensional reaction rate (3) if we take the planar burning velocity, $S_{L}$, as a scale for the velocity, while $S_{a}=\sqrt{2 B L e Z e^{-2} \alpha} \exp \left(-E / 2 R T_{e}\right)$ is the asymptotic value of the velocity of the planar flame calculated in the limit $Z e \gg 1$ [Zeldovich et al., 1985]. For large but finite values of $Z e$, the accurate calculation of the factor $u_{p}=S_{L} / S_{a}$ requires the solution of a one-dimensional eigenvalue problem. For large $Z e$ the eigenvalue $u_{p}$ is, according to [Bush \& Fendell 1970], of the form

$$
\begin{aligned}
S_{L} / S_{a} & =u_{p}(Z e, L e, \gamma) \\
& =1+\Lambda_{1}(L e, \gamma) / Z e+\ldots
\end{aligned}
$$

Nevertheless, in this study, instead of using this asymptotic formula, the factor $u_{p}$ was calculated numerically by a shooting method.

In Eqs. (1) and (2) the velocity of the frame of reference $V=U / S_{L}$ could be chosen arbitrarily. However, in order to prevent the flame from leaving the computational domain, it is convenient to attach the frame of reference to some point that moves with the flame. The nondimensional velocity $V(t)$ of the flame relative to the solid wall was determined, as in [Kurdyumov et al., 2000], by posing a constant temperature, equal to a value $\theta_{*}<1$, at a fixed point $\left(x_{*}, y_{*}\right)$ of the reference frame:

$$
\theta\left(x_{*}, y_{*}\right)=\theta_{*} .
$$

The velocity $V(t)$ thus obtained determines the upstream motion of the frame of reference, and thereby of the flame front, relative to the wall. Or, more precisely, the motion of the point of the flame at a distance $y=y_{*}$ from the wall, where $\theta$ reaches first a given value $\theta_{*}$. In cases of unsteady flame propagation $V(t)$ will be an important feature of the time-dependent flame behavior. If, after an initial transient period, the flame propagates with a constant velocity, the temperature distribution becomes steady in the frame of reference attached to the front.

There is some freedom for our choice of the reference point, $x_{*}, y_{*}$, where $\theta=\theta_{*}$; for example, we can always choose $x_{*}=0$; however, the value of $y_{*}$ should be chosen out of the quench layer, whose size may be expected to scale with $\delta_{L}$, but not too large to be away from the flame front region. The introduction of the exact value of $u_{p}$ in (3) leads to $V=1$ for the steady propagation of planar flames, when $A=0$.

\section{Numerical Method}

Equations (1) and (2) were discretized using finite difference second-order, three-point approximations for space derivatives. A time marching, explicit, procedure using first- or second-order (predictorcorrector) discretization was adopted. Due to the strong variation of the reaction rate term, the time step was chosen sufficiently small as to ensure numerical stability. No significant differences were found in the numerical results when comparing the results obtained from first-order or second-order time discretization.

Taking into account that the temperature in the reference point is constant, the finite difference temperature equation written for this point, when a first-order approximation is used in time, becomes

$$
\left(x_{*}, y_{*}\right):\left(V+K y_{*}\right) M_{x} \theta=M_{x x} \theta+M_{y y} \theta+\omega\left(\theta_{*}, Y_{*}\right) \text {, }
$$

where $Y_{*}$ is the fuel concentration in the reference point; $M_{x} \theta, M_{x x} \theta$ and $M_{y y} \theta$ are the finite-difference representations of the space derivatives, where six points around $\left(x_{*}, y_{*}\right)$ are involved. In this study, the finite difference condition (9) applied for every time step was used in two different ways.

In the first one, $V$ was calculated using (9) for a fixed value of $K$. Then, the resulting value of $V$ was used to compute explicitly the variables for the rest of the grid points at the next time level. Since $V(t)$ represents the velocity of the frame of reference chosen in the above mentioned manner, the 
frame of reference remains attached to a point of the flame with $\theta=\theta_{*}$. This procedure gives the real time-dependent evolution of the flame motion, with the distribution at each time level having a physical meaning as long as a point exists when $\theta=\theta_{*}$ at $\left(x_{*}, y_{*}\right)$ and $\partial \theta /\left.\partial x\right|_{\left(x_{*}, y_{*}\right)} \neq 0$. If the problem has steady multiple solutions, only the stable ones can be obtained in such a way, since the unstable ones will evolve in time to either a stable or to an unsteady one.

In the second method, the finite difference condition (9) was applied to calculate $K$ for a given value of $V$. Then, the value $K$ was used to calculate the variables for the rest of the grid points at the next time level. This procedure, essentially iterative, required a much smaller time step, which now plays the role of an iterative parameter. Only terminal, steady, distributions have a physical sense. As shown below, when this method is used steady unstable solutions are obtained, which are unreachable using the first method.

There are some considerations that must be taken into account in order to properly select $\theta_{*}$ and $y_{*}$. Evidently, $\theta_{*}$ in (8) must be less than the maximum flame temperature and greater than the minimum, fresh mixture, temperature. The value $\theta_{*}=0.5$ was used, and results checked to ensure that $\partial \theta /\left.\partial x\right|_{\left(x_{*}, y_{*}\right)} \neq 0$. We also checked that the chosen reference point is out of the quench layer near the wall. We found that, specially in unsteady cases, it is better to choose a point near the forefront of the flame. The oscillations will be smaller and the instantaneous flame velocity will remain close to the time average velocity.

In cases of stable steady solutions, the first and second procedures lead to the same results, which satisfy the finite difference equations, for definite values of $V$ or $K$. The calculations were carried out for a finite computational domain and the reference point, $x_{*}$, can be chosen so that $x_{*}=0$. The criterion for a steady distribution was $\max _{i, j, k}|\hat{\theta}-\theta| / \tau<$ $10^{-5}$, where $\hat{\theta}$ and $\theta$ are the values of the temperature at current and previous time levels, respectively, and $\tau$ is the time step.

\section{Results}

The main remaining parameters in the problem of Eqs. (1)-(6) are $K$, the Karlovitz number, Le, the Lewis number of the fuel, and $Z e$, the nondimensional activation energy, which are large compared with unity. This fact is taken into account in the planar flame stability analyses, see for example [Clavin, 1985], to show that $L e$ and $Z e$ appear coupled as $l e=Z e(L e-1)$. However, we shall not analyze in this paper the distinguished limit $Z e \rightarrow \infty$ with le finite, when the flame reaction layer shrinks to a surface, but solve numerically the problem for various $L e$ and a finite value of $Z e$, typically $Z e=15$.

We shall begin with the description of the stationary solutions of the problem (1)-(6), which provide, if the solution is stable, the constant flame front velocity and flame front structure, in terms of $K$ for various values of $L e$ and a fixed large value of $Z e$. The unsteady solutions will be described in Sec. 4.2 .

We should notice that $K$, when small compared with unity, represents the ratio of the thickness, $\delta_{L}=\alpha / S_{L}$, of the planar flame and the size, $l_{F}=S_{L} / A$, of the flame front region. Thus if $K \ll 1$, the weakly curved flames are, therefore, prone to instabilities, when $l e=Z e(L e-1)$ lies out of the interval, $-2<l e<10.5$, of planar flame stability [Barenblatt et al., 1962]. Within this interval of $l e$, we can expect $V \rightarrow 1$ in the limit $K \rightarrow 0$. The effects of flame curvature become strong in the flame front region for values of $K$ of order unity, when $\delta_{L}$ and $l_{F}$ are of the same order, and may stabilize the front there, but not downstream where the stretch effects are weak.

\subsection{Stationary solutions for the flame front}

We give the results of the numerical description of the stationary, or time independent, solutions of the problem of the structure of the flame in the flame front region. A typical structure is shown in Fig. 1, calculated for $L e=1$ and $K=0.067$ leading to $V=0.052$ - corresponding to the onset of flashback, or to the critical value of the Karlovitz number, below which we have flashback. In the figure we show, with solid lines, the iso-reaction rate contours, and with dashed lines, the isotherms; in this case, for $L e=1$, the temperature rises monotonously from its upstream value, $\theta=0$, to the asymptotic value, the adiabatic flame temperature $\theta=1$, downstream from the flame and away from the wall.

We also show in Figs. 2 and 3 the flame front structure at the onset of flashback for $L e=0.8$ and $L e=1.2$. In the first case, the temperature 


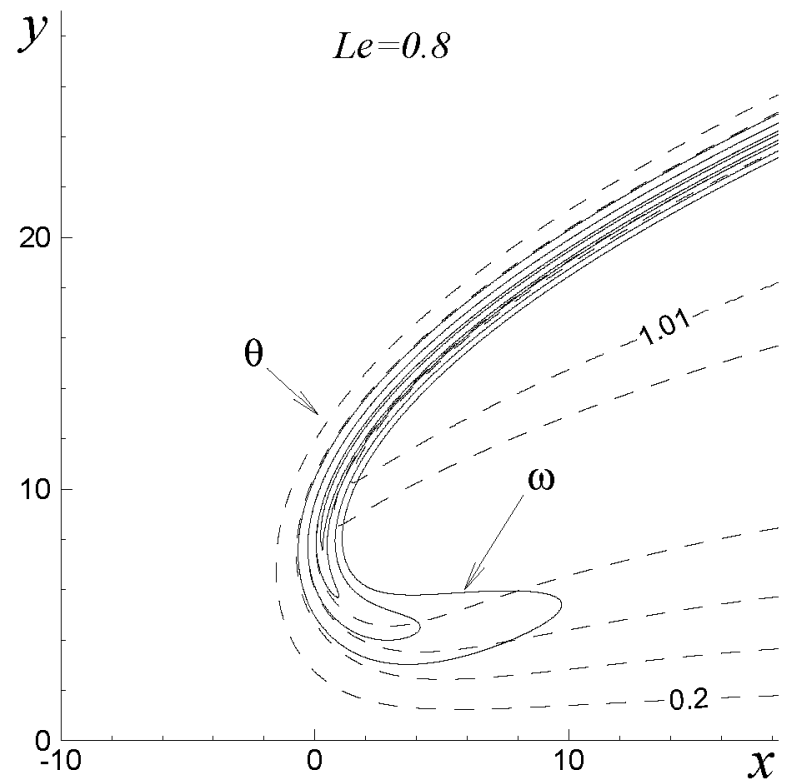

Fig. 2. Isotherms (dashed lines: $\theta$ at intervals $0.2,0.4,0.6$, $0.8,1,1.005$ and 1.001 ) and reaction rate contours (solid lines: $\omega=0.01,0.1,1$ and 2) calculated at the onset of the flashback, $U / S_{L}=0.092, L e=0.8$ and $Z e=15$. The calculated value of the Karlovitz number is $K=0.094$.

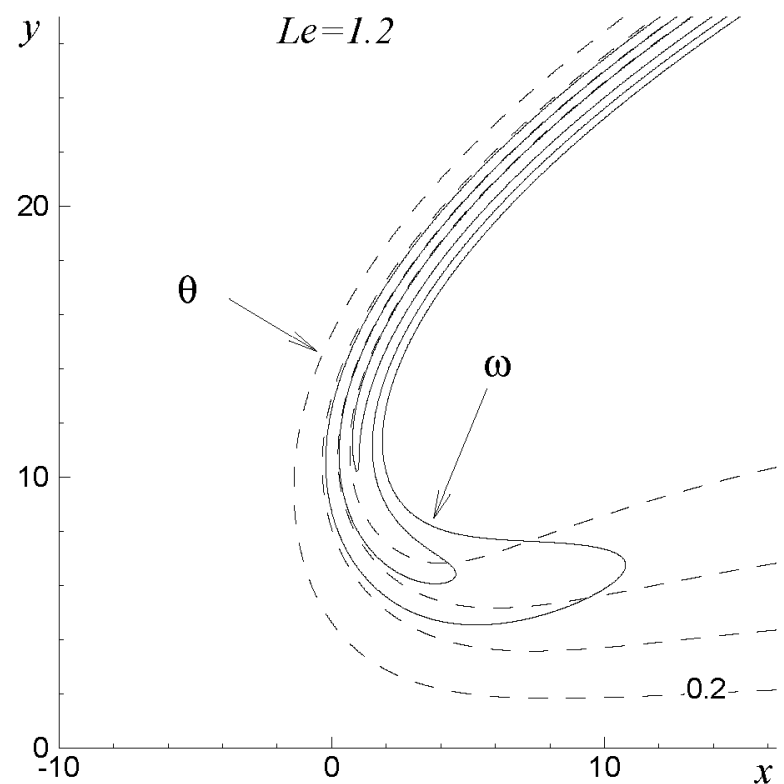

Fig. 3. Isotherms (dashed lines: $\theta$ at intervals 0.2, 0.4, 0.6, 0.8 ) and reaction rate contours (solid lines: $\omega=0.01,0.1,1$ and 2) calculated at the onset of the flashback, $U / S_{L}=0$, $L e=1.2$ and $Z e=15$. The calculated value of the Karlovitz number is $K=0.043$.

$\theta$ rises, due to curvature and nonunity Lewis number effects, to values above $\theta=1$ in the flame front; while in the second case the temperature behind the flame in the flame front region is significantly lower than $\theta=1$.

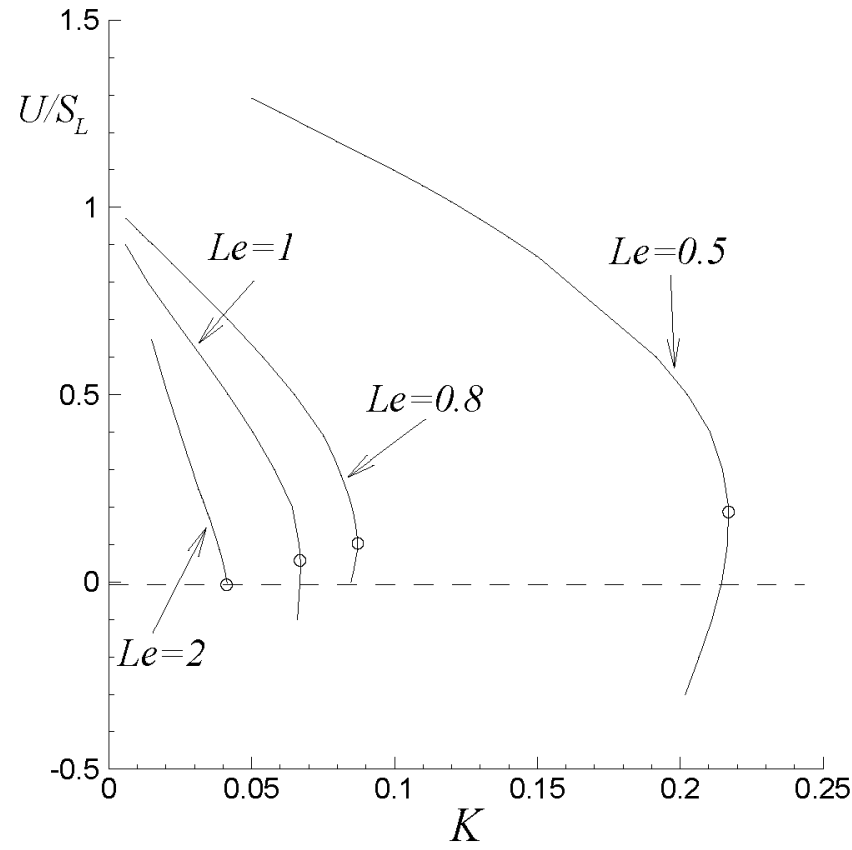

Fig. 4. Nondimensional steady flame front velocity (solid lines) as a function of Karlovitz number for various $L e$ and $Z e=15$. Circles - critical points for flashback.

The stationary values of the flame front velocity are shown in Fig. 4 as a function of $K$ for various values of $L e$; the calculated values are mainly shown for the cases $V>0$ when there is flashback. Observe that some curves, including the curve calculated for $L e=1$, are double valued. The points for onset of flashback are shown in this figure with circles; no solutions exist with $V>0$ for values of $K>K_{c}$. The stable solutions correspond to the points above the circles; the branches of unstable solutions, below the circles, were calculated using the second method described in the previous section. Even without rigorous stability analysis, some numerical tests indicate that the solutions corresponding to the lower branches are unstable; starting time dependent calculations from any point of this branch the upper branch was obtained, after a transient response. The stabilization of the solutions shown in Fig. 4, when they are stable, is due to the strong stretch of the flame associated with the velocity gradient in the flame front region.

\subsection{Unsteady flame front behavior}

In spite of the stabilization due to stretching of the flame by the velocity gradient, when the Lewis number is below the planar flame stability value $L e=1-2 / Z e$, the flame tail downstream of 


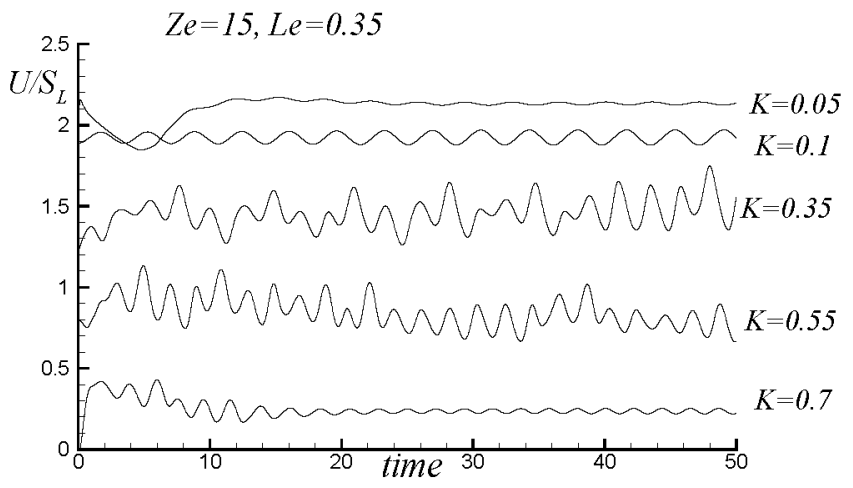

Fig. 5. Time evolution of the nondimensional flame front velocity for $L e=0.35, Z e=15$ and different Karlovitz numbers.

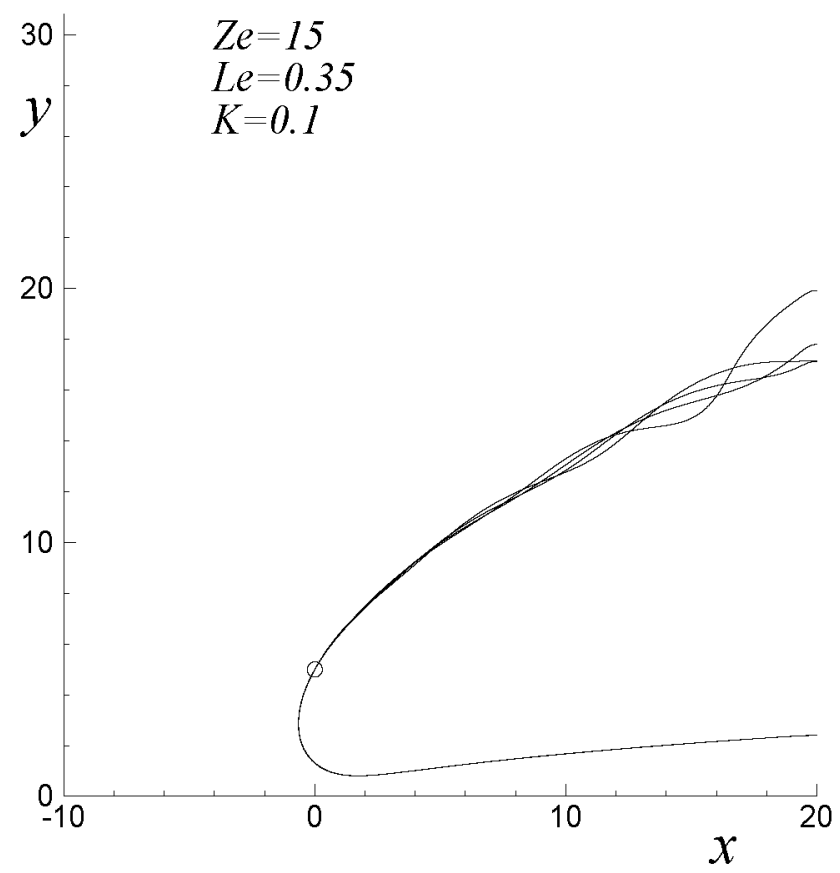

Fig. 6. Isotherms $\theta=0.5$ at different instants calculated for $K=0.1, Z e=15$ and $L e=0.35$. Reference point is shown by circle.

the flame front region, where the effective flame stretch becomes small, loses stability and becomes unsteady. If the loss of stability occurs away from the front region it does not lead to significant time variation in the front velocity and flame front structure. However, this is not the case for small enough values of the Lewis number, when the flame front structure is no longer steady.

The results of the unsteady calculations shown in Fig. 5 give the time evolution of the flame front velocity, for $L e=0.35$ and several values of $K$. Notice, first, the moderately large values of $V(t)$ for the smaller values of $K$, due to the nonunity Lewis num- ber effects associated with flame curvature. Notice, also, that for small values of $K$ the time variations in front velocity are small, because the oscillations will travel downstream along the curved flame before reaching high amplitudes. At large values of $K$ the heat losses to the wall decrease the flame front speed, in spite of the strong curvature effects. The resulting front dynamics loses again its chaotic behavior.

When the flame becomes entirely unstable, it does not move as a whole unchanging structure. Fig. 6 shows the isotherm $\theta=0.5$ at different instants calculated for $K=0.1$ and $L e=0.35$. The reference point is shown in this figure with a circle. Notice that the velocity of this point with respect to the wall is not constant, as shown in Fig. 5. One can see that amplitude of the oscillations of the flame increases downstream, but near the flame front region the flame looks more rigid; the flame front structure has a pulsating motion. This is further illustrated in Fig. 7 where the position of the points with $\theta=0.5$ are shown at three different distances from the cold wall. These figures show that, to facilitate the description of the flame front dynamics, the point of attachment of the frame of reference must lie in the flame front region.

Shown in Fig. 8 are the isotherms and reaction rate iso-contours, plotted for a given time, calculated for $L e=0.32, Z e=15$ and $K=0.1$. One can see that the flame has a very pronounced cellular structure with zones where the flame appears to be quenched. The oscillation of the velocity propagation, for these conditions, shown in Fig. 9, are chaotic; the time-average flame propagation velocity exceeds significantly the planar flame velocity.

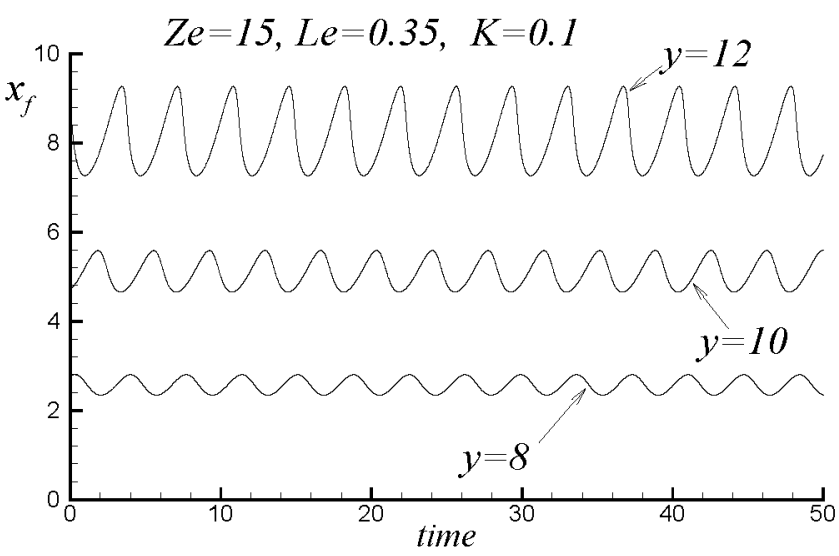

Fig. 7. Time evolution of the points of the flame, $x=x_{f}$, located in the intersection of the isotherm $\theta=0.5$ and lines with $y=8,10$ and 12 . 


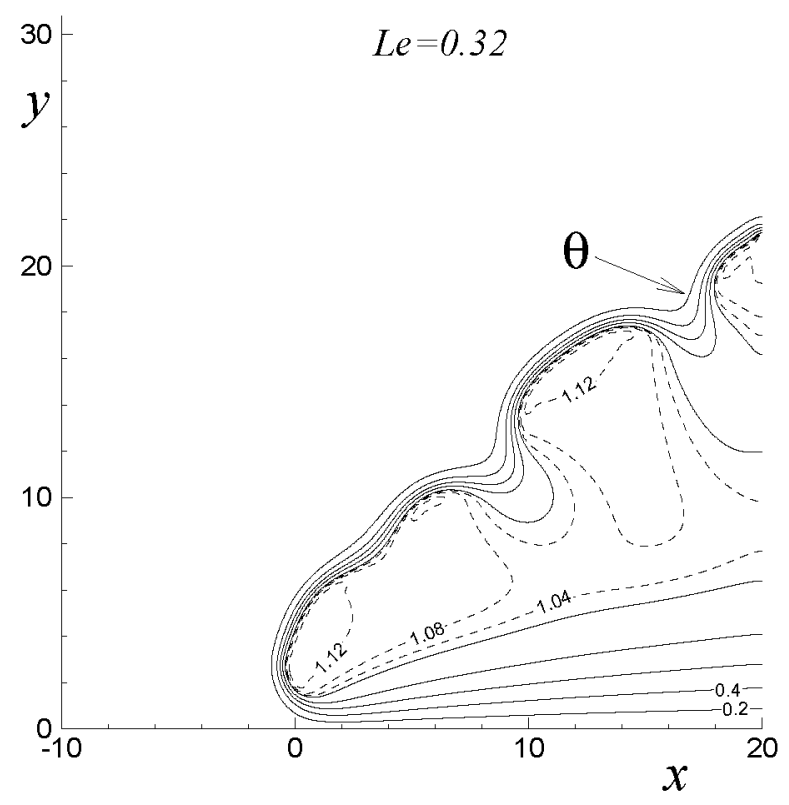

(a)

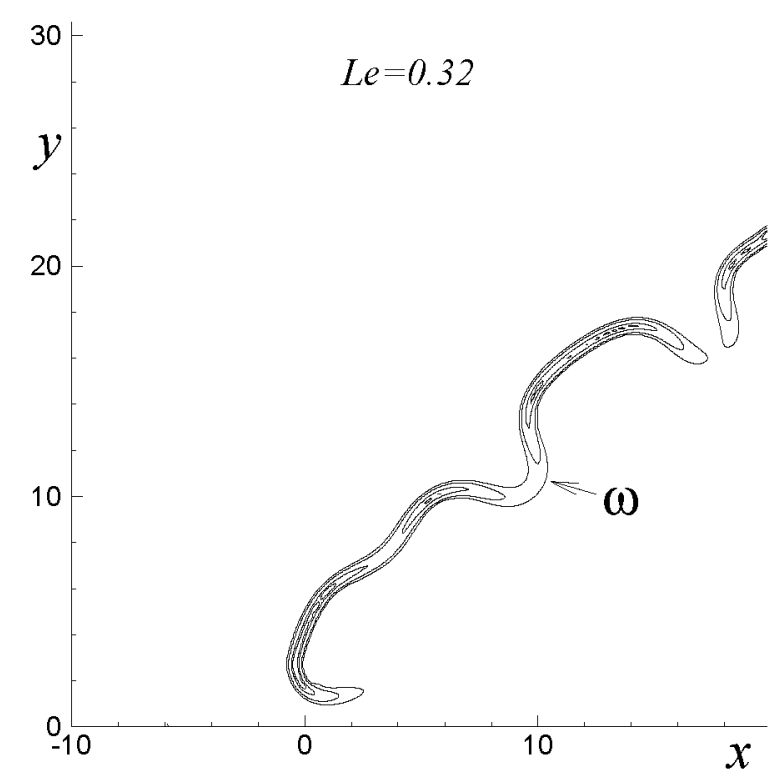

(b)

Fig. 8. Typical unsteady cellular flame structure calculated for $L e=0.32, Z e=15$ and $K=0.1$. (a) Isotherms (solid lines: $\theta$ at intervals $0.2, \theta_{\min }=0.2, \theta_{\max }=1$; dashed lines: $\theta=1.04,1.08$ and 1.12); (b) reaction rate contours $(\omega=0.25,1,5$ and 8).

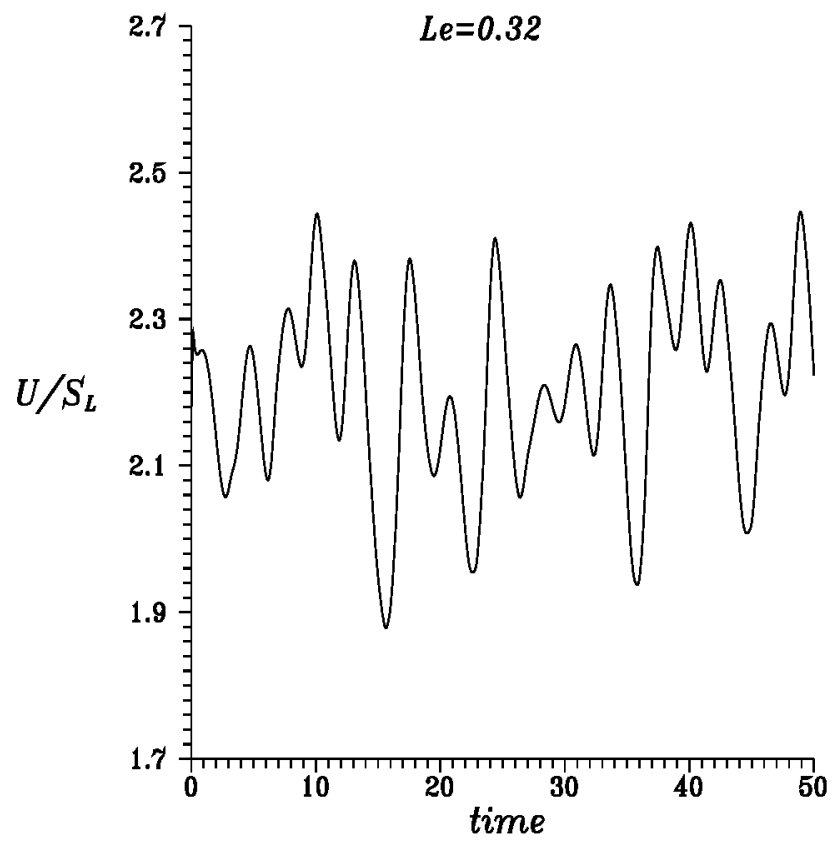

Fig. 9. Time evolution of the nondimensional flame front velocity calculated for $L e=0.32, Z e=15$ and $K=0.1$.

\section{Concluding Remarks}

We have presented some of the results of the numerical solution of the problem of Eqs. (1)-(6). These have been carried out to describe the flame structure of a reaction front propagating, against the flow of a reactive mixture, along the layer, near a cold wall, where the flow velocities are of the order of $S_{L}$, the speed of propagation of the planar flame.

The analysis is applicable to the description of the conditions that lead to flashback, or upstream flame propagation relative to the wall, when, as it is the case of the flow in the premixing tube of LP or LPP burners, the flow velocity is large compared with $S_{L}$. In this case the flame front encounters a flow field of constant shear $A$, corresponding to the base of the boundary layer. The structure of the flame front region determines the propagation speed of the front.

In our analysis we have accounted for important effects of the Lewis number of the fuel, which are enhanced by the curvature of the flame due to the velocity gradient, and also to the heat losses to the wall. We have not taken into account effects of the variations with temperature of the diffusivities and of the density changes. Although these effects may have a significant influence on the results, we should notice that in LP and LPP burners the ratio of the flame and initial temperatures is limited to values below 2.5 .

With the thermal-diffusive approximation, used here, the front propagation speed $U$, measured with $S_{L}$ is a function, $U / S_{L}=V(K, L e)$, of the Karlovitz number, $K=A \alpha / S_{L}^{2}$, and the Lewis number $L e$. 
We may expect the ratio $U / S_{L}$ to be weakly dependent on the nondimensional activation energy $Z e$, for realistic values, $Z e \gg 1$. See [Kurdyumov et al., 2000]. In addition, we may expect the results not to be strongly dependent on the kinetics, when the planar flame velocity $S_{L}$ is used as a scale for $U$ and $S_{L}^{2} / \alpha$ is used as a scale for $A$.

One important result of the calculations is that flashback, which corresponds to positive values of $U$, is not possible for values of the Karlovitz number $K$ above a critical value $K_{c}(L e)$, which increases with decreasing values of $L e$; the resulting values of $K_{c}$ are not of order unity, as suggested by Lewis and von Elbe [1951], but roughly of order $1 / Z e$. Of the two branches of the curve $V(K, L e)$, formed for values of $K<K_{c}$, the one with lower values of $V$ is unstable; even though, we have not carried out a linear stability analysis of the stationary solutions of the problem, which is not an easy task. The stability analysis carried out for the planar flame, see [Barenblatt et al., 1962] or [Clavin, 1985], indicate that the domain of instability lies outside the interval $-2<l e<10.5$.

The numerical computations, which we have carried out for the unsteady evolution of the flame front, show that for values of the Lewis numbers $L e$ such that $l e<-2$ the time-varying front propagation velocity, which may reach values significantly large compared with $S_{L}$, has a chaotic behavior if $K$ is neither very small compared with $K_{c}$ nor close to $K_{c}$. This chaotic behavior appears to evolve from solutions which show an apparently periodic $V(t)$, with a trailing flame showing a traveling chaotic structure.

\section{Acknowledgments}

Both authors have the privilege of many years of fruitful discussion and exchange of ideas with Prof. M. G. Velarde. We are happy to contribute this paper, showing some of the complicated dynamic behavior of flames, in honor of Prof. Velarde's sixtieth birthday. We look forward for a long continued association with him in the future. The work presented here has been supported by the Spanish Ministry of Science and Technology under Project BFM 2001-3691.

\section{References}

Barenblatt, G. I., Zeldovich, Y. B. \& Istratov, A. G. [1962] "On diffusional-thermal stability of a laminar flame," Zh. Prikl. Mekh. Thkh. Fiz. 2, 21-26.

Bush, W. B. \& Fendell, F. E. [1970] "Asymptotic analysis of laminar flame propagation for general Lewis numbers," Combust. Sci. Technol. 1, 421-428.

Clavin, P. [1985] "Dynamic behavior of premixed flame fronts in laminar and turbulent flows," Progr. Energy Combust. Sci. 11, 1-60.

Clavin, P. [1994] "Premixed combustion and gasdynamics," Ann. Rev. Fluid Mech. 26, 321-352.

Clavin, P. [2000] "Dynamics of combustion fronts in premixed gases: From flames to detonations," Proc. Combust. Instit. 28, 569-585.

Kurdyumov, V. N., Fernández, E. \& Liñán, A. [2000] "Flame flashback and propagation of premixed flames near a wall," Proc. Combust. Instit. 28, 1883-1889.

Kurdyumov, V. N. \& Fernández, E. [2002] "Lewis number effect on the propagation of premixed laminar flames in narrow open ducts," Combust. Flame $\mathbf{1 2 8}$, $382-394$.

Lee, S. T. \& Tien, J. S. [1982] "A numerical analysis of flame flashback in a premixed laminar system," Combust. Flame 4, 273-285.

Lewis, B. \& von Elbe, G. [1943] "Stability and structure of Burner flames," J. Chem. Phys. 11, 75-97.

Lewis, B. \& von Elbe, G. [1951] Combustion, Flames and Explosions of Gases (Academic Press, NY).

Mallens, R. M. M. \& de Goey, L. P. H. [1998] "Flashback of laminar premixed methane/air flames on slit and tube burners," Combust. Sci. Tech. 136, 41-54.

Sivashinsky, G. [1983] "Instabilities, pattern formation, and turbulence in flames," Ann. Rev. Fluid Mech. 15, $179-199$.

Thibaut, D. \& Candel, S. [1998] "Numerical study of unsteady turbulent premixed combustion: Application to flashback simulation," Combust. Flame 113, 53-65.

von Karman, T. \& Millán, G. [1953] "Thermal theory of a laminar flame front near a cold wall," Proc. Combust. Instit. 4, 173-178.

Williams, F. A. [1985] Combustion Theory, 2nd edition (The Benjamin/Cummings Publishing Company).

Zeldovich, Ya. B., Barenblatt, G. I., Librovich, V. B. \& Mahkviladze, G. M. [1985] The Mathematical Theory of Combustion and Explosions (Consultants Bureau, $\mathrm{NY}$ ). 